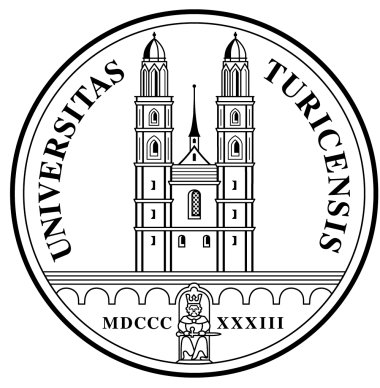

Institute for Empirical Research in Economics

University of Zurich

Working Paper Series

ISSN 1424-0459

Working Paper No. 74

\title{
Evolution of Portfolio Rules in Incomplete Markets
}

Thorsten Hens and Klaus Reiner Schenk-Hoppé

October 2001 (Revised Version) 


\title{
Evolution of Portfolio Rules in Incomplete Markets*
}

\author{
Thorsten Hens \\ Klaus Reiner Schenk-Hoppé \\ Institute for Empirical Research in Economics \\ University of Zurich, Switzerland
}

October 16, 2001

\begin{abstract}
The paper considers the evolution of portfolio rules in markets with stationary returns and endogenous prices. The ultimate success of a portfolio rule is measured by the wealth share the rule is eventually able to conquer in competition with other portfolio rules. We give necessary and sufficient conditions for portfolio rules to be evolutionary stable. In the case of i.i.d. returns we identify a simple portfolio rule to be the unique evolutionary stable strategy. Moreover we demonstrate that mean-variance optimization is not evolutionary stable while the CAPM-rule always imitates the best portfolio rule and survives.
\end{abstract}

JEL-Classification: D52, D81, D83, G11.

Keywords: portfolio theory, evolutionary finance, incomplete markets.

${ }^{*}$ We are grateful to Igor Evstigneev and seminar participants at Ecofin, NHH-Bergen, University of Munich, ETH-Zurich, and the Stockholm School of Economics. Thorsten Hens wants to thank Sandra Güth for collaboration on this topic in an early stage. Klaus Reiner Schenk-Hoppé acknowledges helpful comments by Jörgen Weibull.

Contact address: thens@iew.unizh.ch, klaus@iew.unizh.ch 


\section{Introduction}

We consider an incomplete asset market with a finite number of assets and a continuum of states. Asset payoffs are given by a stationary process in discrete time. Assets are short lived but identically "re-born" in every period. A finite number of portfolio rules manage capital by iteratively re-investing on the given asset market. There are no transaction costs. Portfolio rules are non-negative vectors of expenditure shares for assets which may depend on the past observations. The set of portfolio rules we consider is not restricted to those generated by expected utility maximization. It may as well include investment rules favored by behavioral finance models. Savings and withdrawals are exogenously determined. In every period in time the available market capital is given by the total payoff of the assets. Portfolio rules compete for this market capital - the endogenous price process is thus a market selection mechanism along which some strategies gain market capital while others lose.

This model has been introduced in the seminal paper of Blume and Easley (1992). It is well suited to analyze the performance of large institutional investors like pension plans, insurance companies or mutual funds. Those institutions are investing on asset markets that do not provide complete insurance against all possible risks. This seems to be the natural case. Savings and withdrawals are exogenously determined because they are the pensions and the indemnities to be payed or they are the liquidity demanded by the clients of the institutions. Institutional investors do have a considerable impact on asset market prices, face relatively small transaction costs and their investment horizon is potentially infinite. Finally it is not clear at all that institutional investors maximize some infinite horizon expected utility function.

The equilibrium notion this model provides is a distribution of market capital (wealth shares) that is invariant under the market selection process. We show that provided there are no redundant assets every invariant distribution of market shares is generated by a monomorphic population, i.e. a collection of traders using the same portfolio rule. We derive a general evolutionary (in-)stability criterion for such monomorphic populations. Roughly speaking a portfolio rule is evolutionary stable if it has the highest exponential growth rate in a population where itself determines market prices. In a sense an evolutionary stable population plays the "best response against itself." From this criterion it follows for example that any population with misspecified beliefs can successfully be invaded by a portfolio rule being based on the maximization of expected utility with rational expectations. Moreover we demonstrate that mean-variance optimization can be invaded by any 
completely diversified portfolio rule while the CAPM-rule is able to always imitate the best portfolio rule and thus survives.

In the i.i.d. case the robustness of the invariant distributions with respect to the innovation of new portfolio rules singles out one portfolio rule that is the unique evolutionary stable strategy, i.e. that drives out any mutations. According to this rule one should divide wealth proportionally to the expected relative payoffs of the assets. This is a very simple explicit formula which is quite easy to apply in actual markets. It even does not require the knowledge of the actual probabilities driving the payoff process. It only requires to compute the sample mean of the payoffs because under the i.i.d. assumption this is clearly an unbiased estimator for the future expected payoffs. The effect of this rule on asset prices is that it equalizes the expected relative returns of all assets. In particular then asset pricing is risk neutral pricing even though the unique evolutionary stable portfolio rule can be thought of as being generated by quite risk avers investors - those having unit relative risk aversion.

In the case of diagonal securities ${ }^{1}$ this strategy boils down to a well-known trading strategy that in this case is known to be the global attractor of the market selection mechanism, or as Blume and Easley (1992) have called it the single survivor. Since with diagonal securities prices do not matter, this rule coincides with the well known rule "betting your beliefs" (Breiman 1961) according to which income should be divided proportionally to the probability of the states. This strategy evidently maximizes the growth rate of wealth. It can be generated by maximizing the expected logarithm of relative returns which in turn is known as the Kelly rule (Kelly 1956).

Our paper extends Blume and Easley's (1992) model to any complete or incomplete payoff structure. In this case market prices matter in the evolution of wealth shares. To overcome this difficulty we exploit the idea of evolutionary stability which so far has not been used in any portfolio theory. However, as in Blume and Easley (1992), we consider an economy with short-lived assets. With regards to possible applications of the theory, this is an unsatisfactory assumption that will have to be generalized in future research. With complete markets, recently, Blume and Easley (2000) and Sandroni (2000) have investigated the case of long-lived assets in an economy with completely rational investors. They show that among all infinite horizon expected utility maximizers those who happen to have rational expectations will eventually dominate the market. This interesting result holds irrespectively of the investors' risk aversion characteristics. The intuition

\footnotetext{
${ }^{1}$ We call a system of securities diagonal when in each state exactly one asset has a non-zero payoff. An example for these are Arrow-securities.
} 
goes that with complete markets agents can place any bets on differences in their expectations so that those with rational expectations will always win in the long run. As Blume and Easley (2000) made perfectly clear this result is ultimately linked to Pareto-efficiency. In our setting we get similar conclusions with respect to rational expectations without relying on Paretoefficiency.

On passing it is worthwhile to mention the relation between the evolutionary portfolio theory literature and the classical finance approach to maximize the expected growth rate of wealth for some exogenously given return process. In a series of papers, Hakansson (1970), Thorp (1971), Algoet and Cover (1988), and Karatzas and Shreve (1998), among others, have explored this maximum growth perspective. Computing the maximum growth portfolio is a non-trivial problem. Even if one restricts attention to i.i.d. returns, when markets are incomplete, there is no explicit solution to this problem. By now numerical algorithms to compute the maximum growth portfolio have been provided by Algoet and Cover (1988) and Cover (1984, 1991). But so far practical decisions are rarely based on these ideas. Our result is interesting in this respect because the simple portfolio rule that we obtain shows that considering the equilibrium consequences of expected growth rate maximization does not make matters more complicated but much easier.

In the next section we present the economic model which has the mathematical structure of a random dynamical system. Then we define the equilibrium concepts and the stability notions, Section 3. In Section 4 we discuss and generalize Blume and Easley's result in the case of incomplete markets. Section 5 presents our main result which will be proved using a series of propositions that are also of independent interest. In Section 6 we analyze the evolutionary fitness of portfolio rules based on mean-variance optimization. We study the issue of under-diversified portfolios, and discuss the implication of the CAPM investment strategy. All proofs are relegated to the Appendix.

\section{The Model}

Time is discrete and indexed by $t$. The possible states of nature are determined in each period in time by the realization of a stationary stochastic process with values in some measurable space $(S, \mathcal{S})$. Let $(\Omega, \mathcal{F}, \mathbb{P}, \theta)$ denote its canonical realization as a metric dynamical system on the path space, i.e. $\Omega$ is the sample path space with representative element $\omega=\left(\ldots, \omega_{-1}, \omega_{0}, \omega_{1}, \ldots\right)$, $\mathcal{F}=\mathcal{S}^{\mathbb{Z}}$ is the corresponding $\sigma$-algebra, $\mathbb{P}$ is the associated probability measure, and $\theta$ is the shift map (defined by $\theta \omega(\cdot)=\omega(1+\cdot)$ ). The family $\theta^{t}$, 
$t \in \mathbb{Z}$ (where $\theta^{t}$ denotes the $t$-times iterate of $\theta$ ) defines a measurable flow on $\Omega$, i.e. $\theta^{t+u}=\theta^{t} \circ \theta^{u}$ for all $u, t \in \mathbb{Z}, \theta^{0}=\mathrm{id}_{\Omega}$, and $\theta$ is measurable and measurably invertible. Stationarity implies $\theta \mathbb{P}=\theta^{-1} \mathbb{P}=\mathbb{P}$. $\omega_{t}$ denotes the state of nature at time $t$, and the sequence of observations up to the end of period $t$ is referred to as $\omega^{t}$. Further let $\mathcal{F}^{t}=\sigma\left\{\omega_{u} \mid u<t\right\}$ denote the information available at the beginning of period $t$. A sequence of random variables $\left(s_{t}\right)_{t \in \mathbb{Z}}$ such that $s_{t}$ is measurable with respect to $\mathcal{F}^{t}$ is called adapted to the filtration $\left(\mathcal{F}^{t}\right)_{t \in \mathbb{Z}}$. By definition, an $\mathcal{F}^{t}$-measurable random variable can only depend on $\omega^{t-1}$.

There are finitely many investors $i=1, \ldots, I$ endowed with wealth $w_{0}^{i}>0$ at time 0 . Assets $k=1, \ldots, K$ with $K \geq 2$, live for one period only but are identically re-born in every period. Their payoffs $A_{t}^{k}(\omega)$ are assumed to be adapted. We make the following assumption.

Assumption 1 For all $t$, (1) $A_{t}^{k}(\omega) \geq 0$ for all $k$ and all $\omega$; (2) for each $k$ there exists a set $\Omega_{k} \in \mathcal{F}$ with $\mathbb{P}\left(\Omega_{k}\right)>0$ such that $A_{t}^{k}(\omega)>0$ for all $\omega \in \Omega_{k}$; and (3) $\sum_{k=1}^{K} A_{t}^{k}(\omega)>0$ for all $\omega$.

This assumption ensures that all assets yield non-negative payoffs in all states of nature, each asset has a strictly positive payoff for a set of states of nonzero measure, and total payoff of all assets is strictly positive in every state.

In each period in time $t$ every investor selects a portfolio $a_{t}^{i}=\left(a_{1, t}^{i}, \ldots, a_{K, t}^{i}\right)$ with values in $\mathbb{R}_{+}^{K}$. $\quad a_{t}^{i}: \Omega \rightarrow \mathbb{R}_{+}^{K}$ is assumed to be adapted. Given the portfolio $a_{t}^{i}$ at time $t$, the investor's wealth in period $t+1$ is given by,

$$
w_{t+1}^{i}=\sum_{k=1}^{K} A_{t}^{k}(\omega) a_{k, t}^{i}
$$

Letting $\rho_{k, t}$ denote the price of asset $k$ in period $t$, then-provided that the agent's wealth is positive - his budget shares are given by,

$$
\lambda_{k, t}^{i}:=\frac{\rho_{k, t} a_{k, t}^{i}}{w_{t}^{i}}
$$

We define the trading strategy of investor $i$ as a sequence of budget shares $\lambda_{t}^{i}=\left(\lambda_{1, t}^{i}, \ldots, \lambda_{K, t}^{i}\right)_{t \geq 0}$. Since $a_{t}^{i}$ and $\rho_{k, t}$ are adapted for all $t$ so is each budget share $\lambda_{t}^{i}$.

Assuming that any investor exhausts his budget in all periods in time, i.e. the portfolio is chosen such that $\sum_{k=1}^{K} \rho_{k, t} a_{k, t}^{i}=w_{t}^{i}$ for all $t \geq 0$, every trading strategy $\lambda_{t}^{i}$ takes values in the unit simplex $\Delta^{K}:=\left\{x \in \mathbb{R}_{+}^{K} \mid \sum_{k=1}^{K} x_{k}=1\right\}$. 
The market-clearing prices are given by,

$$
\rho_{k, t}=\frac{1}{\bar{a}_{t}^{k}} \sum_{i=1}^{I} \lambda_{k, t}^{i} w_{t}^{i}
$$

where $\bar{a}_{t}^{k}>0$, assumed to be adapted, is the total supply of asset $k$ at time $t$. For the market selection process to be well defined, we need to guarantee that equilibrium prices $\rho_{t}$ are always positive. A sufficient condition for this is that some trading strategy with positive initial wealth is completely mixed, i.e. it has only strictly positive budget shares in every period in time and in every state of nature. We make the following assumption.

Assumption 2 In every market there is some trading strategy $\lambda_{t}^{i}$ with positive initial wealth $w_{0}>0$ that is completely mixed, i.e. $\lambda_{t}^{i}(\omega) \in \operatorname{int} \Delta^{\mathrm{K}}$ for all $\omega \in \Omega$.

It is clear from Assumption 1 and equation (1) that any completely mixed trading strategy with positive wealth in a period of time maintains a positive wealth for all future periods. Thus any trader with strictly positive wealth in one period in time will have strictly positive wealth in all subsequent periods in time, if he pursues a completely mixed trading strategy.

Taking into account how equilibrium prices are determined, we obtain a recursive formula for the total wealth of each consumer. Consumer $i$ 's wealth in period $t+1$ is given by,

$$
w_{t+1}^{i}=\sum_{k=1}^{K} A_{t}^{k}(\omega) \bar{a}_{t}^{k} \frac{\lambda_{k, t}^{i} w_{t}^{i}}{\sum_{j=1}^{I} \lambda_{k, t}^{j} w_{t}^{j}}
$$

and the total market wealth in period $t+1, w_{t+1}:=\sum_{i} w_{t+1}^{i}$, can be equated as,

$$
w_{t+1}=\sum_{i=1}^{I} w_{t+1}^{i}=\sum_{k=1}^{K} A_{t}^{k}(\omega) \bar{a}_{t}^{k}
$$

Note that in the definition of next period wealth we have assumed that no investor saves or withdraws any money along the process. Our results carry over to the case of saving rates which are constant over time and identical among investors.

The prices of the assets, normalized by the market wealth, are given by,

$$
q_{k, t}:=\frac{\rho_{k, t}}{w_{t}}=\sum_{i=1}^{I} \lambda_{k, t}^{i} r_{t}^{i}
$$


i.e. the normalized price is a convex combination of the trading strategies for asset $k$ over the wealth shares of investors. $q_{k, t}$ is adapted.

¿From (3) and (4) we obtain a recursive formula for the evolution of the wealth (or market) shares $r_{t}^{i}:=w_{t}^{i} / w_{t}$,

$$
r_{t+1}^{i}=\sum_{k=1}^{K} \frac{A_{t}^{k}(\omega) \bar{a}_{t}^{k}}{\sum_{l=1}^{K} A_{t}^{l}(\omega) \bar{a}_{t}^{l}} \frac{\lambda_{k, t}^{i} r_{t}^{i}}{\sum_{j=1}^{I} \lambda_{k, t}^{j} r_{t}^{j}}
$$

Finally we define the relative payoff of asset $k$ as,

$$
R_{t}^{k}(\omega):=\frac{A_{t}^{k}(\omega) \bar{a}_{t}^{k}}{\sum_{l=1}^{K} A_{t}^{l}(\omega) \bar{a}_{t}^{l}}
$$

Assumption 3 (1) The relative payoff of each asset is a stationary random variable, i.e. $R_{t}^{k}(\omega)=R^{k}\left(\theta^{t} \omega\right)$.

(2) All strategies are stationary, i.e. $\lambda_{t}^{i}(\omega)=\lambda^{i}\left(\theta^{t} \omega\right)$.

For any given set of stationary adapted strategies $\left(\lambda^{i}\right)=\left(\lambda^{i}\right)_{i=1, \ldots, I}$ the evolution of wealth shares (6) can be written as,

$$
r_{t+1}=f\left(\theta^{t} \omega, r_{t}\right)
$$

where

$$
f_{i}\left(\theta^{t} \omega, r\right)=\sum_{k=1}^{K} R^{k}\left(\theta^{t} \omega\right) \frac{\lambda_{k}^{i}\left(\theta^{t} \omega\right) r^{i}}{\sum_{j=1}^{I} \lambda_{k}^{j}\left(\theta^{t} \omega\right) r^{j}}
$$

We refer to equation (7) as the market selection process in the following.

The dynamical description of the market selection process employs the framework of a random dynamical system (Arnold 1998). (7) generates a random dynamical system in the following sense. Let $f(\omega):=f(\omega, \cdot): \Delta^{I} \rightarrow$ $\Delta^{I}$, and define

$$
\varphi(t, \omega, r):= \begin{cases}f\left(\theta^{t-1} \omega\right) \circ \ldots \circ f(\omega) r & \text { for } t \geq 1 \\ r & \text { for } t=0 \\ f\left(\theta^{t} \omega\right)^{-1} \circ \ldots \circ f\left(\theta^{-1} \omega\right)^{-1} r & \text { for } t \leq-1\end{cases}
$$

In words, $\varphi(t, \omega, r)$ is the vector of wealth shares of all investors at time $t$ when the initial distribution of wealth shares is $r$ and the sequence of realizations of states $\omega$ prevails.

$f^{-1}(\omega)$ is well-defined for all $\omega \in \Omega$ if all strategies are completely mixed and there are no redundant assets. In this case $\varphi$ is defined for all $t \in \mathbb{Z}$; otherwise $t \in \mathbb{N}$. In the following we restrict attention to the case in which $f$ is invertible. 
The family of maps $\varphi(t, \omega, r)$ is a random dynamical system on the unit simplex $\Delta^{I}$. That is, $\varphi: \mathbb{Z} \times \Omega \times \Delta^{I} \rightarrow \Delta^{I},(t, \omega, r) \mapsto \varphi(t, \omega, r)$ is a $\mathcal{B}(\mathbb{Z}) \otimes \mathcal{F} \otimes \mathcal{B}\left(\Delta^{I}\right), \mathcal{B}\left(\Delta^{I}\right)$ measurable ${ }^{2}$ mapping such that $\varphi(0, \omega)=\operatorname{id}_{\Delta^{I}}$ and $\varphi(s+t, \omega)=\varphi\left(t, \theta^{s} \omega\right) \circ \varphi(s, \omega)$ for all $s, t \in \mathbb{Z}$, and $\omega \in \Omega$. We refer the reader to the monograph by Arnold (1998) for any additional information.

It is important to emphasize that the random dynamical system generated by (7) depends on the trading strategies pursued by the investors. That is, for any set of strategies $\left(\lambda^{i}\right)$ there is a unique random dynamical system generated by $(7)$.

\section{Evolutionary Stability}

In this section we introduce the stability concepts needed to analyze the long term behavior of the wealth shares under the market selection process (7).

Given a random dynamical system for a set of stationary and adapted trading strategies $\left(\lambda^{i}\right)$ one is particularly interested in those wealth shares that evolve in a stationary fashion over the infinite time-horizon. Here we restrict ourselves to deterministic distributions of wealth shares that are fixed under the market selection process $(7) .^{3}$ To specify this notion, we recall the definition of a deterministic fixed point in the framework of random dynamical systems. Let a set of strategies $\left(\lambda^{i}\right)$ be given, and denote by $\varphi$ the associated random dynamical system.

Definition $1 \bar{r} \in \Delta^{I}$ is called a (deterministic) fixed point of $\varphi$ if for all $\omega \in \Omega$,

$$
\bar{r}=\varphi(1, \omega, \bar{r})(\equiv f(\omega, \bar{r})) .
$$

The distribution of wealth shares $\bar{r}$ is said to be invariant under the marketselection process ( 7$)$.

Condition (10) is equivalent to $\bar{r}=\varphi(t, \omega, \bar{r})$ for all $t$ and all $\omega$.

The assumption of stationarity discharges us from using the common "almost surely," because any condition or result with this additional restriction can be transferred into a "for all $\omega$ " statement by restricting the space $\Omega$ to an invariant subset of full $\mathbb{P}$-measure. This claim only holds in that generality because time is discrete here.

If $r^{i}=0$, then $\varphi^{i}(t, \omega, r)=0$ by (7). Therefore, in any set of trading strategies each unit vector in $\Delta^{I}$ (i.e. each corner) is a fixed point. In words,

\footnotetext{
${ }^{2} \mathcal{B}$ denotes the Borel $\sigma$-algebra, and $\mathcal{B}\left(\Delta^{I}\right):=\mathcal{B}\left(\mathbb{R}^{I} \cap \Delta^{I}\right)$ is the trace $\sigma$-algebra.

${ }^{3}$ See e.g. Schenk-Hoppé (2001) for applications of the general concept of a random fixed point in economic growth.
} 
the state in which one investor possesses the entire market does not change over time.

We are particularly interested in those invariant distributions of wealth shares which are stable under the market selection process. Roughly speaking, stability means that small perturbations of the initial distribution of wealth shares do not have a long-run effect. If an invariant distribution of wealth shares is stable, all sample paths starting in a neighborhood of this distribution at time zero and the sample path of the invariant distribution of the wealth shares are asymptotically identical. We will need different notions of stability; they are defined as follows.

Definition 2 An invariant distribution of wealth shares $\bar{r} \in \Delta^{I}$ is called (locally) stable, if there exists a random open set $U(\omega)$ containing $\bar{r}$ such that for all $\omega, \lim _{t \rightarrow \infty}\|\varphi(t, \omega, r)-\bar{r}\|=0$ for all $r \in U(\omega)$.

$U(\omega)$ is a random open set, if it is an open set for all $\omega$ and $\left\{\omega \mid\left(\Delta^{I} \backslash U(\omega)\right) \cap\right.$ $G \neq \emptyset\} \in \mathcal{F}$ for all open sets $G$, cf. Arnold (1998, Chap. 1.6).

Given a locally stable invariant distribution of wealth shares $\bar{r}$, then any initial distribution of wealth shares in a small neighborhood of $\bar{r}$ is asymptotically identical to $\bar{r}$ as time tends to infinity.

It is straightforward to see that this notion of stability does not make sense on the level of individual investors in general. For example, suppose $r$ is an invariant distribution of wealth shares for the random dynamical system $\varphi$ associated to the pair of strategies $\left(\lambda^{1}, \lambda^{2}\right)$. Then $\bar{r}_{\alpha}:=\left(r^{1},(1-\alpha) r^{2}, \alpha r^{2}\right)$ is invariant for the random dynamical system, say $\varphi_{\alpha}$, on $\Delta^{3}$ associated to $\left(\lambda^{1}, \lambda^{2}, \lambda^{2}\right)$ for all $\alpha \in[0,1]$. However, $\bar{r}_{\alpha}$ can never (even if $r$ is locally stable for $\varphi$ ) be locally stable for the random dynamical system $\varphi_{\alpha}$ on $\Delta^{3}$.

We will therefore interpret a distribution of wealth shares $r$ as a distribution over populations of players where all players within each group play the same strategy. Thus the wealth share $r^{i}$ denotes that fraction of the total market wealth belonging to the players of strategy $\lambda^{i}$. Under this assumption it is clear that all strategies $\lambda^{i}$ are different from each other, i.e. $\lambda^{i} \neq \lambda^{i^{\prime}}$ for all $i, i^{\prime}, i \neq i^{\prime}$.

The above definition refers to the stability of a distribution of wealth in a population with given strategies. However, one would also like to have a notion of stability in the case that new strategies occur on the market. We first note that the structure of the market selection process (7) implies the following extension property. Let $\left(\lambda^{i}\right)_{i \in \mathcal{I}}, \mathcal{I}=\{1, \ldots, I\}$, be any set of completely mixed strategies. Suppose $\bar{r}$ is an invariant distribution of wealth shares for the corresponding random dynamical system on $\Delta^{I}$. Then for any set $\left(\lambda^{j}\right)_{j \in \mathcal{J}}, \mathcal{J}=\{1, \ldots, J\}$ with $J \geq 0(\mathcal{J}=\emptyset$, if $J=0)$, of strategies, 
$(\bar{r}, 0, \ldots, 0) \in \Delta^{I+J}$ ( $J$-times zero) is an invariant distribution of wealth shares for the random dynamical system on $\Delta^{I+J}$ associated to the set of strategies $\left(\left(\lambda^{i}\right)_{i \in \mathcal{I}},\left(\lambda^{j}\right)_{j \in \mathcal{J}}\right)$.

Given a set of strategies $\left(\lambda^{i}\right)_{i \in \mathcal{I}}$ another set of strategies $\left(\lambda^{j}\right)_{j \in \mathcal{J}}$ is called new, if with strictly positive probability (1) $\lambda^{j} \neq \lambda^{i}$ for all $j \in \mathcal{J}, i \in \mathcal{I}$ and (2) $\lambda^{j} \neq \lambda^{j^{\prime}}$ for all $j, j^{\prime} \in \mathcal{J}, j \neq j^{\prime}$, i.e. adding a set of new strategies yields a market in which no redundant strategies are present.

Definition 3 An invariant distribution of wealth shares $\bar{r} \in \Delta^{I}$ is called evolutionary stable, if for all $J \geq 0,(\bar{r}, 0, \ldots, 0) \in \Delta^{I+J}$ is stable for all sets of strategies $\left(\left(\lambda^{i}\right)_{i \in \mathcal{I}},\left(\lambda^{j}\right)_{j \in \mathcal{J}}\right)$ with $\left(\lambda^{j}\right)_{j \in \mathcal{J}}$ being new.

A strategy is called evolutionary stable, if the invariant distribution of wealth shares $1 \in \Delta^{1}$ is evolutionary stable.

For each evolutionary stable distribution of wealth shares there exits an entry barrier (a random variable here) below which an arbitrary number of new strategies do not drive out the incumbent players. Any perturbation, if sufficiently small, does not change the long-run behavior of the distribution of wealth shares. The market selection process asymptotically leaves the mutants with no wealth share while the market is shared between the incumbents as unchanged. As discussed above, we do not allow for redundant strategies to be introduced.

Unlike in evolutionary game theory, this notion of stability refers to the distribution of wealth shares and not to the set of strategies. It may well be the case that for a given set of strategies there are two different stable invariant distributions of wealth shares one of which being evolutionary stable and the other not.

Finally, we define a corresponding local stability criterion.

Definition 4 An invariant distribution of wealth shares $\bar{r} \in \Delta^{I}$ is called locally evolutionary stable, if for all $J \geq 0$ there exists a random variable $\delta(\omega)>0$ such that $(\bar{r}, 0, \ldots, 0) \in \Delta^{I+J}$ is locally stable for all sets of strategies $\left(\left(\lambda^{i}\right)_{i \in \mathcal{I}},\left(\lambda^{j}\right)_{j \in \mathcal{J}}\right)$ with $\left(\lambda^{j}\right)_{j \in \mathcal{J}}$ being new and $\min _{i \in \mathcal{I}} \max _{j \in \mathcal{J}} \| \lambda^{i}(\omega)-$ $\lambda^{j}(\omega) \|<\delta(\omega)$ for all $\omega$.

A strategy is called locally evolutionary stable, if the invariant distribution of wealth shares $1 \in \Delta^{1}$ is locally evolutionary stable.

A locally evolutionary stable distribution of wealth shares is evolutionary stable with respect to local mutations. That is, the strategies that can be pursued by all mutants are limited to small deviations from existing strategies.

We can now turn to the study of the long-run outcome of the market selection process. 


\section{Blume and Easley's result revisited}

We briefly outline Blume \& Easley's (1992) findings in the framework of random dynamical systems theory, extending their result to a model with stationary portfolio rules, a general set of states of nature, and an ergodic process determining the state of nature.

Analogous to Blume and Easley (1992) we assume that the payoffs of the assets are diagonal. In our more general setting that is, there exists a measurable partition $\left(\Omega_{k}\right)_{k=1, \ldots, K}$ of $\Omega$ into sets with strictly positive measure such that $R^{k}(\omega)=1$ if and only if $\omega \in \Omega_{k}$. (Recall that $R^{k}(\omega) \in\{0,1\}$ for diagonal securities.) Due to this property we can unambiguously denote the relative payoffs in the market selection process (7) at time $t$ by $R\left(\theta^{t} \omega\right)$.

A trading strategy of investor $i$ is a stationary (not necessarily adapted here) random variable $\lambda^{i}: \Omega \rightarrow \Delta^{K}, \lambda_{k}^{i}\left(\theta^{t} \omega\right)$ being her wealth share invested in asset $k$ at time $t$. By the diagonal payoff structure we may unambiguously denote by $\lambda_{\theta^{t} \omega}^{i}$ the wealth share invested in that asset $k$ with $\theta^{t} \omega \in \Omega_{k}$.

Due to the assumption of diagonal payoffs (7) simplifies to,

$$
r_{t+1}^{i}=R\left(\theta^{t} \omega\right) \frac{\lambda_{\theta^{t} \omega}^{i} r_{t}^{i}}{\sum_{j=1}^{I} \lambda_{\theta^{t} \omega}^{j} r_{t}^{j}}
$$

The evolution of the ratio of the wealth shares of any two investors, say $i$ and $j$, using completely mixed trading strategies can then be written as,

$$
\frac{r_{t+1}^{i}}{r_{t+1}^{j}}=\frac{\lambda_{\theta^{t} \omega}^{i}}{\lambda_{\theta^{t} \omega}^{j}} \frac{r_{t}^{i}}{r_{t}^{j}}
$$

because the normalized asset price, $\sum_{j=1}^{I} \lambda_{\theta^{t} \omega}^{j} r_{t}^{j}$, cancels out for diagonal securities.

Fix any initial wealth shares $r_{0}^{i}>0$ and $r_{0}^{j}>0$. Then the asymptotic behavior of the ratio of the two wealth shares is given by,

$$
\lim _{T \rightarrow \infty} \frac{1}{T} \ln \frac{r_{T}^{i}}{r_{T}^{j}}=\lim _{T \rightarrow \infty} \frac{1}{T} \sum_{t=0}^{T-1} \ln \frac{\lambda_{\theta^{t} \omega}^{i}}{\lambda_{\theta^{t} \omega}^{j}}=\mathbb{E} \ln \frac{\lambda_{\omega}^{i}}{\lambda_{\omega}^{j}}
$$

for almost all sample paths $\omega$. The equality on the far right-hand side of the last equation holds by the Birkhoff ergodic theorem. The expected value is finite if the strategies are completely mixed.

Consequently we obtain that along almost any sample path $\omega$,

$$
\lim _{T \rightarrow \infty} \frac{1}{T} \ln \frac{r_{T}^{i}}{r_{T}^{j}}>0 \text { if and only if } \mathbb{E} \ln \lambda_{\omega}^{i}>\mathbb{E} \ln \lambda_{\omega}^{j}
$$


The equation on the left-hand side implies that for almost all $\omega, \ln r_{T}^{i}(\omega) \geq$ $T \varepsilon+\ln r_{T}^{j}(\omega)$ for all sufficiently large $T$, where $\varepsilon>0$. Since $\ln r_{T}^{i}(\omega) \leq 0$ for all $T$ and all $\omega, \ln r_{T}^{j}(\omega) \rightarrow-\infty$ as $T \rightarrow \infty$. Thus, we find that $r_{T}^{i}(\omega) \rightarrow 1$ almost surely.

This result implies the following asymptotic behavior of the market selection process. Those investors who are closest to maximizing the expected logarithm of the wealth shares will eventually dominate the market. This result holds regardless of the initial distribution of wealth shares in the population. However, note that even in the case discussed here the surviving population depends on the strategies present in the population.

The best choice an investor can make in a period $t$ is to set $\lambda_{\theta^{t} \omega}^{i}=1$ if and only if $\theta^{t} \omega \in \Omega_{k}$. However, this requires knowledge of the state $\omega_{t}$ prior to the revelation of the random draw at time $t$. The strategy is therefore not adapted. If the only information available to investors at time $t$ is given by $\mathcal{F}^{t}$, their strategies have to be adapted, i.e. $\lambda^{i}\left(\theta^{t} \omega\right)$ has to be $\mathcal{F}^{t}$-measurable (and thus can only depend on $\omega^{t-1}$ at time $t$ ) for every $i$.

Among all adapted strategies the optimal portfolio rule is given by the random variable $\lambda_{\omega} \in \Delta^{K}$ that maximizes $\mathbb{E}\left(\ln \lambda_{\omega} \mid \omega^{-1}\right)$, the expected logarithm of $\lambda_{\omega}$ contingent on the observed past $\omega^{-1}=\left(\omega_{-1}, \omega_{-2}, \ldots\right)$.

For instance if the ergodic process determining the state of nature is a Markov process, then the optimal portfolio rule is one that depends only on the last observation $\omega_{t-1}$. All further information from the observed history is not helpful in that case. The optimal strategy is given by $\lambda_{k}(\omega)=$ $\int 1_{\Omega_{k}}\left(\omega_{0}\right) P\left(d \omega_{0}, \omega_{-1}\right)=P\left(\Omega_{k}, \omega_{-1}\right)$ where $P\left(\omega_{0}, \omega_{-1}\right)$ denotes the transition probability. If the state space $S$ is finite, $\lambda_{k}(\omega)=P\left(k, \omega_{-1}\right)$ (the transition probability), where $\omega_{-1}$ is the state observed in the preceding period in time.

The case considered in Blume and Easley (1992) is derived by assuming that the random draw is i.i.d. and a finite set of states $s=1, \ldots, S$ with $\mathbb{P}\{\omega \mid$ $\left.\omega_{t}=s\right\} \equiv p_{s}>0$. Then the state $\omega_{t}$ is independent of the observed history up to time $t, \omega^{t-1}$. In this case the past does not contain any information on the future and thus it is optimal to play a simple trading strategy, i.e. the budget shares are deterministic and fixed for all times. The right-hand side of equation (11) then becomes

$$
\sum_{s=1}^{S} p_{s} \ln \lambda_{s}^{i}>\sum_{s=1}^{S} p_{s} \ln \lambda_{s}^{j}
$$

Consequently — as in Blume and Easley (1992) — we obtain that those investors who are closest to maximizing the expected logarithm of the budget shares $\lambda_{s}$ (under the distribution of the one-period random draw) will eventually dominate the market. The strategy maximizing the expected logarithm 
of the budget shares $\lambda_{s}$ is "betting your beliefs", i.e. $\lambda_{s}=p_{s}$ for all $s$.

As already pointed out above, if this strategy is present in the population then it is the unique long-run outcome of the market-selection process. Equation (11) is an absolute fitness criterion in the sense that it is independent of the population under consideration. With diagonal securities maximizing the expected logarithm conditional on the past observation means maximizing the growth rate of wealth in any population.

\section{Main Results}

In this section we show that there exists a unique evolutionary stable strategy in any incomplete market. This portfolio rule has an explicit representation. Moreover, we can show that any other investment strategy is not even locally evolutionary stable.

As pointed out above, we restrict our analysis to deterministic invariant wealth shares. It is first proved that these fixed points correspond to monomorphic populations if there are no redundant assets. We then derive necessary conditions for the (local) stability and instability of such stationary wealth shares in the general case in which portfolio rules are adapted. The central mathematical tool is the multiplicative ergodic (or Oseledets's) theorem.

These conditions are used to single out one particular portfolio rule $\lambda^{\star}$ as being evolutionary stable if all investors use simple portfolio rules, i.e. strategies that are independent of past observations. In the i.i.d. case we prove that $\lambda^{\star}$ is the only evolutionary stable portfolio rule in the set of all adapted strategies. We further show that any other portfolio rule can be driven out even by portfolio rules arbitrary close to it, i.e. it is not even locally evolutionary stable.

With a general payoff matrix we can no longer benefit from the cancellation of prices in the evolution of relative wealth shares (an essential property that was used in Section 4) and there are some important conceptual differences to the case of diagonal securities. In contrast to that case there is no longer an absolute fitness criterion for the survival of trading strategies. The growth rate of any trading strategy now depends essentially on the population in which it lives. Restricting attention to the question of local stability of deterministic invariant distributions circumvents these problems and is still sufficient to single out a unique evolutionary stable trading strategy. Before presenting the main results of the paper, we derive two auxiliary results that are also of independent interest.

We make the following assumption throughout the following. 
Assumption 4 There are no redundant assets.

Assumption 4 requires that for any two portfolios $a^{1}, a^{2}: \Omega \rightarrow \Delta^{K}$ with $a^{1}(\omega) \neq a^{2}(\omega)$ on a set of strictly positive measure, $\sum_{k} R^{k}(\omega)\left(a_{k}^{1}(\omega)-\right.$ $\left.a_{k}^{2}(\omega)\right) \neq 0$ on a set of strictly positive measure. That is different portfolios cannot generate the same payoff stream almost surely.

We have already noted that every distribution of wealth shares in which the players of only one strategy possess the entire market wealth is invariant under the market selection process (and is a deterministic fixed point). Moreover, if there are no redundant assets there is also a converse to this observation as the following result shows.

Proposition 1 Only one strategy can have strictly positive wealth in every population of strategies with a (deterministic) invariant distribution of wealth shares.

In Proposition 1 all deterministic invariant distributions of wealth shares are characterized. We next derive a sufficient condition for the stability of such fixed points under Assumptions 1-4. The following result is central to the proof of the main results.

Proposition 2 Let the state be determined by an ergodic process. Given any set of adapted strategies $\left(\lambda^{i}\right)$. The invariant distribution of wealth shares $\bar{r}=e_{n}$ being concentrated on the players of the completely mixed $n$-th strategy is

(i) stable, if

$$
\mathbb{E} \ln \left(\sum_{k=1}^{K} R^{k}(\omega) \frac{\lambda_{k}^{i}(\omega)}{\lambda_{k}^{n}(\omega)}\right)<0 \quad \text { for all } i \neq n ;
$$

(ii) unstable, if

$$
\mathbb{E} \ln \left(\sum_{k=1}^{K} R^{k}(\omega) \frac{\lambda_{k}^{i}(\omega)}{\lambda_{k}^{n}(\omega)}\right)>0 \quad \text { for some } i \neq n .
$$

The conditions in Proposition 2 have the following interpretation. In a situation in which the prices of all assets are determined by the stationary portfolio rule $\lambda^{n}$ we can measure the exponential growth rate of other, competing portfolio rules. If the invariant distribution of wealth shares $\bar{r}=e_{n}$ is stable then the strategy $\lambda^{n}$, which completely determines the prices, has a higher growth rate in a neighborhood of this distribution of market shares 
than all other portfolio rules in the population. However, if there is at least one strategy that has a higher growth rate for these prices, $\bar{r}=e_{n}$ is unstable and the $\lambda^{n}$-player does not reobtain total market wealth after a slight deviation from the possessing-everything situation. From the condition (13) any monomorphic population playing $\lambda^{n}(\omega)$ can be successfully invaded by a trader maximizing the expected logarithm given the prices $\lambda^{n}(\omega)$. In particular any incumbent who maximizes expected utility according to incorrect beliefs $\tilde{p}$ will be driven out by the trader maximizing expected logarithmic utility according to the correct beliefs $p$.

The quantities on the left-hand side of (12) resp. (13) are the Lyapunov exponents of the linearization of the random dynamical system generated by (7) (the market selection mechanism) at the corner of the simplex $\Delta^{I}$, i.e. the fixed point in which only one investor holds total market wealth. The eigenspaces correspond to the vertices of the simplex.

Note that stability resp. instability of a status quo strategy $\lambda^{n}$ is determined by pair-wise comparisons with all other strategies in the population. This is due to the fact that the linearization at each corner of the simplex $\Delta^{I}$ is a diagonal matrix. Evolutionary stability of a strategy $\lambda^{n}$ thus means that condition (i) in Proposition 2 is satisfied for all adaptive strategies $\lambda^{i} \neq \lambda^{n}$. There is no need to check for stability of $\lambda^{n}$ in any possible pool of strategies.

The main result of our paper is based on the observation that, allowing for all possible mutations, only one particular strategy satisfies the necessary condition for stability derived in Proposition 2.

Denote by

$$
g\left(\lambda^{i}, \lambda^{n}\right):=\mathbb{E} \ln \left(\sum_{k=1}^{K} R^{k}(\omega) \frac{\lambda_{k}^{i}(\omega)}{\lambda_{k}^{n}(\omega)}\right) .
$$

the exponential growth rate appearing on the left-hand side of (12) and (13).

Suppose there is some portfolio rule $\lambda^{\star}$ such that $g\left(\lambda, \lambda^{\star}\right)<0$ for all $\lambda \neq \lambda^{\star}$. Then we obtain

$$
g\left(\lambda^{\star}, \lambda\right)=\mathbb{E} \ln \left(\sum_{k=1}^{K} R^{k}(\omega) \frac{\lambda_{k}^{\star}(\omega)}{\lambda_{k}(\omega)}\right) \geq \mathbb{E} \ln \left(\sum_{k=1}^{K} R^{k}(\omega) \frac{\lambda_{k}(\omega)}{\lambda_{k}^{\star}(\omega)}\right)^{-1}>0 .
$$

by the Jensen inequality because $R^{k}(\omega)$ is a probability measure on $\{1, \ldots, K\}$. Summarizing this result, we can state the following corollary.

Corollary 1 Suppose all investors employ completely mixed portfolio rules. Then existence of a portfolio rule that is evolutionary stable (i.e. stable in any pool of strategies) implies that all other portfolio rules cannot be evolutionary stable. 
The task is to show that there is an evolutionary stable portfolio rule and to give an explicit formula for this rule.

Assume momentarily that the state of nature is determined by an i.i.d. process. Then the relative payoff of asset $k$ at time $t$ is determined by the state of nature in the respective period, i.e. $R^{k}\left(\theta^{t} \omega\right)=R^{k}\left(\omega_{t}\right)$. The probability measure on the sample path space $\Omega=S^{\mathbb{Z}}$ is given by the product measure $\mathbb{P}=\mu^{\mathbb{Z}}$, where $\mu$ is the distribution of the state in each period in time. In this case,

$$
g\left(\lambda^{i}, \lambda^{n}\right)=\int_{S^{\mathbb{N}}} \int_{S} \ln \left(\sum_{k=1}^{K} R^{k}(s) \frac{\lambda_{k}^{i}\left(\omega^{-1}\right)}{\lambda_{k}^{n}\left(\omega^{-1}\right)}\right) \mu(d s) \mu^{\mathbb{N}}\left(d \omega^{-1}\right),
$$

where $\omega^{-1}=\left(\omega_{-1}, \omega_{-2}, \ldots\right)$ is the observed history at time $t=0$.

If investors do not base their portfolio decision on any past observations (for instance because they know that the state is determined by an i.i.d. process), their portfolio rule is determined by a simple trading strategy, i.e. a deterministic vector of budget shares $\left(\lambda(\omega) \equiv \lambda \in \Delta^{K}\right)$. Under this assumption the above equation simplifies to

$$
g\left(\lambda^{i}, \lambda^{n}\right)=\int_{S} \ln \left(\sum_{k=1}^{K} R^{k}(s) \frac{\lambda_{k}^{i}}{\lambda_{k}^{n}}\right) \mu(d s)
$$

If we want to check for evolutionary stability of a simple strategy $\lambda^{n}$ in the set of all simple strategies, we need to show that condition (i) in Proposition 2 holds for all $\lambda^{i} \in \Delta^{K}$ with $\lambda^{i} \neq \lambda^{n}$, i.e. the term in (16) is strictly less than zero. Note that for fixed $\lambda^{n} \in \operatorname{int} \Delta^{\mathrm{K}}$ (or for fixed $\lambda^{i} \in \operatorname{int} \Delta^{\mathrm{K}}$ ) (16) defines a map from $\Delta^{K}$ to $\mathbb{R}$. This observation is also true for both conditions in Proposition 2 if the state process is ergodic and investors only employ simple strategies.

The situation is more complicated if, for instance, the status quo strategy $\lambda^{n}$ depends on the observation $\omega^{-1}$. Then the quantity (15) also depends on the past - albeit the payoffs of all assets are independent of the past. The stability condition in Proposition 2 depends on the past only through the investor's belief that past observations matter in choosing a portfolio.

If we want to check for evolutionary stability of a strategy $\lambda^{n}$ in this general case it suffices prove the following. For every possible past $\omega^{-1}$ and all deterministic vectors $\lambda^{i} \in \Delta^{K}$ it holds

$$
\int_{S} \ln \left(\sum_{k=1}^{K} R^{k}(s) \frac{\lambda_{k}^{i}}{\lambda_{k}^{n}\left(\omega^{-1}\right)}\right) \mu(d s) \leq 0
$$


and the inequality is strict on a set of strictly positive $\mathbb{P}$-measure. Thus we are back to check stability for simple strategies. And the above equation also defines a map from $\Delta^{K}$ to $\mathbb{R}$.

To check for the absence of evolutionary stability of a strategy $\lambda^{n}\left(\omega^{-1}\right)$ it is sufficient to show that for every possible past $\omega^{-1}$ there exists a deterministic vector $\lambda^{i} \in \Delta^{K}$ such that

$$
\int_{S} \ln \left(\sum_{k=1}^{K} R^{k}(s) \frac{\lambda_{k}^{i}}{\lambda_{k}^{n}\left(\omega^{-1}\right)}\right) \mu(d s) \geq 0
$$

and the inequality is strict on a set of strictly positive $\mathbb{P}$-measure. This defines a map $\omega^{-1} \mapsto \lambda^{i}$. The above inequality implies that the (instability) condition (ii) in Proposition 2 is fulfilled for $\lambda^{i}\left(\omega^{-1}\right)$. However, it is important to point out that one has to make sure that the resulting map is measurable. This is true without any further assumptions, for instance, if $\{s\} \in \mathcal{S}$ for all $s \in S$. Then every past $\omega^{-1} \in \mathcal{F}^{0}$.

Let us finally discuss the Markovian case in which the state of nature is determined by a Markov process with transition probability $P$. We find

$$
g\left(\lambda^{i}, \lambda^{n}\right)=\int_{S^{\mathbb{N}}} \int_{S} \ln \left(\sum_{k=1}^{K} R^{k}\left(s, \omega_{-1}\right) \frac{\lambda_{k}^{i}\left(\omega^{-1}\right)}{\lambda_{k}^{n}\left(\omega^{-1}\right)}\right) P\left(d s, \omega_{-1}\right) \mathbb{P}^{-1}\left(d \omega^{-1}\right)
$$

where $\mathbb{P}^{-1}$ is the distribution of the sample path $\omega^{-1} \in S^{\mathbb{N}}$.

If investors only employ Markovian strategies, i.e. $\lambda\left(\omega^{-1}\right)=\lambda\left(\omega_{-1}\right)$ depends only on the state of nature revealed in the preceding period, then (17) simplifies to

$$
g\left(\lambda^{i}, \lambda^{n}\right)=\int_{S} \int_{S} \ln \left(\sum_{k=1}^{K} R^{k}(s, u) \frac{\lambda_{k}^{i}(u)}{\lambda_{k}^{n}(u)}\right) P(d s, u) \mu(d u)
$$

where $\mu$ is the stationary distribution of the state of nature in each period. The (absence of) evolutionary stability of a strategy can be checked analogously to the procedure explained for the i.i.d. case.

We have the following result.

Theorem 1 Let the state be determined by an ergodic process. Suppose investors only employ simple strategies, i.e. $\lambda(\omega) \equiv \lambda \in \Delta^{K}$. Then the simple strategy $\lambda^{\star}$ defined by,

$$
\lambda_{k}^{\star}=\mathbb{E} R^{k}(\omega),
$$

for $k=1, \ldots, K$ is evolutionary stable, and no other strategy is locally evolutionary stable. 
The portfolio rule $\lambda^{\star}$ divides wealth according to the expected relative payoffs of the assets. For a given asset market of the structure discussed in this paper, the strategy is very simple to compute; it requires a minimum of easily accessible information.

Let us consider the case in which the state of nature can only take finitely many values $s=1, \ldots, S$ in detail. Under the assumption of stationarity $\mathbb{P}\left\{\omega \mid \omega_{t}=s\right\} \equiv p_{s}>0$ for all $s$. Therefore, the portfolio rule $\lambda^{\star}$ in Theorem 1 becomes $\lambda_{s}^{\star}=\sum_{s=1}^{S} p_{s} R^{k}(s)$. It is straightforward to see that we reobtain the result by Blume and Easley (1992, Section 3) in the case of diagonal securities. In this case, $\lambda^{\star}$ corresponds to the Kelly rule of "betting one's beliefs."

The assumption in Theorem 1 that all investors employ simple strategies even though the state is determined by a general ergodic process can be criticized. However, recall that even if the state is i.i.d. the past observations can enter into the market selection mechanism (and thus in the condition for local (in)stability) through investors' beliefs. That is, if a trader adopts a proper adapted portfolio rule then the past matters. Note that the stability criteria in Proposition 2 require an integration over the entire history of the process because the strategies may depend on the past observations.

We have the following general result in the i.i.d. case.

Theorem 2 Let the state be determined by an i.i.d. process. Then $\lambda_{k}^{\star}=$ $\mathbb{E} R^{k}(\omega), k=1, \ldots, K$, is the only evolutionary stable portfolio rule.

Moreover, if $\mathcal{S}$ is the power set of the set of states $S$, then we find that all other completely mixed adapted strategies are not even locally evolutionary stable.

The technical assumption that $\mathcal{S}$ is the power set of the set of states $S$ is fulfilled, for instance, if $S$ is countable (or finite) and $\mathcal{S}$ is the Borel $\sigma$ field. We need this condition to ensure measurability of a strategy that is constructed in the proofs of the next two results to ensure that no portfolio rule different to $\lambda^{\star}$ can be locally evolutionary stable.

Theorem 3 generalizes this result to the Markovian case. The proof of Theorem 3 is a straightforward extension of the proofs of Theorems 1 and 2 and is therefore omitted.

Theorem 3 Let the state be determined by a Markov process. Then the adaptive strategy $\lambda^{\star}$ defined by,

$$
\lambda_{k}^{\star}\left(\omega_{-1}\right)=\mathbb{E}\left(R^{k}(\omega) \mid \omega_{-1}\right),
$$

for $k=1, \ldots, K$ is the only evolutionary stable portfolio rule. 
Moreover, if $\mathcal{S}$ is the power set of the set of states $S$, then we find that all other completely mixed adapted strategies are not even locally evolutionary stable.

The strategy $\lambda^{\star}$ can be interpreted as a Nash equilibrium in the following way. For simplicity we restrict the discussion to the i.i.d. case. Recall the definition of the function $g$ in $(16) . g(\alpha, \beta)$ measures the asymptotic exponential growth rate of a strategy $\alpha$ in a population in which all asset prices are determined by strategy $\beta$. Using Proposition 2, the assertion of Theorem 1 can be stated as:

For all $\alpha \neq \lambda^{\star}$,

$$
\begin{aligned}
g\left(\lambda^{\star}, \lambda^{\star}\right) & >g\left(\alpha, \lambda^{\star}\right) \text { and; } \\
g(\alpha, \alpha) & <g(\beta, \alpha) \text { for some } \beta \text { in every neighborhood of } \alpha .
\end{aligned}
$$

That is to say $\lambda^{\star}$ is the unique symmetric Nash equilibrium in a game with payoff function $g$. Moreover, $\lambda^{\star}$ is also a strict Nash equilibrium. Therefore $\lambda^{\star}$ is the unique evolutionary stable strategy in the sense of Maynard Smith and Price (1973).

\section{Mean-Variance Optimization}

In this section we analyze the evolutionary fitness of portfolio rules based on mean-variance optimization. For clarity of presentation we restrict the analysis to simple strategies.

The mutual fund theorem states that given all investors build portfolios according to the mean-variance-criterion, then every investor will hold a combination of the riskless asset and the market portfolio in any capital market equilibrium. Even though it is very questionable whether indeed all investors use mean-variance-optimization, investing a big share of wealth in the market portfolio is a very common behavior.

We extend our previous model by incorporating a strategy that enables an investor to buy the market portfolio. This extension relates our model to the classical $\mathrm{CAPM}^{4}$ results.

It is well known that in practice mean-variance portfolios are often under diversified, i.e. they typically put positive weight on very few assets only. To cure this defect it is then usually suggested to modify the mean-variance portfolio by devoting some positive but small share of the budget on every asset in

\footnotetext{
${ }^{4}$ See also Sciubba (1998) for an analysis of CAPM-trading rules in the original Blume and Easley (1992) setup with diagonal securities.
} 
the portfolio, ensuring that the portfolio is completely mixed. We show in the next section that this commonly used "quick fix" of the under-diversification problem is indeed an improvement of the mean-variance portfolio.

\subsection{The CAPM strategy}

Consider an investor, say $\gamma$, who wants to buy a fraction of the market portfolio which is the vector of total stock of the assets. In our model each asset is supplied in one unit. Phrased in terms of budget shares, to buy the market portfolio, an investor has to divide his wealth proportional to the asset prices, i.e.,

$$
\lambda_{k, t}^{\gamma}=\frac{\rho_{k, t}}{\sum_{l=1}^{K} \rho_{l, t}} \equiv q_{k, t}
$$

where $k=1, \ldots, K$. This trading strategy depends on the equilibrium prices in the current period. An investor who buys the market portfolio has therefore to give a demand function to the auctioneer. This calls for an extension of our previous analysis.

Suppose all other investors pursue simple trading strategies $\lambda^{i} \in \Delta^{K}$, $i=1, \ldots, I, i \neq \gamma$. Then the market-clearing condition becomes,

$$
q_{k, t}=\sum_{i \neq \gamma} \lambda_{k}^{i} r_{t}^{i}+q_{k, t} r_{t}^{\gamma}
$$

and thus

$$
q_{k, t}=\frac{1}{1-r_{t}^{\gamma}} \sum_{i \neq \gamma} \lambda_{k}^{i} r_{t}^{i}
$$

The evolution of the market wealth of the CAPM investor can be equated as

$$
r_{t+1}^{\gamma}=\sum_{k=1}^{K} R^{k}\left(\theta^{t} \omega\right) \frac{\lambda_{k, t}^{\gamma} r_{t}^{\gamma}}{q_{k, t}}=\sum_{k=1}^{K} R^{k}\left(\theta^{t} \omega\right) r_{t}^{\gamma}=r_{t}^{\gamma}
$$

Summarizing our findings we can state the following result.

Proposition 3 The wealth share of a CAPM investor is constant in any population in which all other players pursue simple strategies. In particular, a CAPM investor will never vanish nor dominate the market.

The intuition behind this result is given by the representation of the normalized market-clearing price in the model with only simple strategies (5). The normalized equilibrium price equals the relative market wealth invested in that asset. If one player dominates the market in the long-run and 
asymptotically own the entire market wealth, the asset price will reflect the trading strategy of this investor. The CAPM investor mimics this strategy because he distributes his wealth according to the relative value of the assets.

From an evolutionary point of view it can be concluded that investing in the market portfolio is a strategy with strong resistance against the market selection mechanism. Hence even though buying the market portfolio may not be in accordance with mean-variance optimization (because not everybody uses it) it is a convenient rule which automatically imitates the most successful trading strategy!

\subsection{Diversification}

We assume that the number of states of nature is finite, $s=1, \ldots, S$.

Corollary 2 Suppose $\hat{\lambda}$ is an under-diversified simple strategy, i.e. $\hat{\lambda}_{k}=0$ for at least one $k$. Denote by $\hat{\lambda}_{k}^{\varepsilon}:=(1-\varepsilon) \hat{\lambda}_{k}+\varepsilon / S, 0<\varepsilon \leq 1$, the corresponding $\varepsilon$-completed strategy. Then $\hat{\lambda}^{\varepsilon}$ is robust against $\hat{\lambda}$-mutants for all sufficiently small $\varepsilon>0$, i.e. the distribution of wealth shares that assigns total wealth to the $\hat{\lambda}^{\varepsilon}$-player is stable in the population $\left(\hat{\lambda}^{\varepsilon}, \hat{\lambda}\right)$.

Even though using the "quick fix" to prevent under-diversification is better than investing according to the under-diversified portfolio rule, it is clear from the main result Theorem 1 , that $\varepsilon$-completed under-diversified simple strategies are not locally stable (if they do not coincide with $\lambda^{\star}$ ). However, we next show that the situation for $\varepsilon$-completed portfolio rules $\hat{\lambda}^{\varepsilon}$ is even worse. Any completely mixed simple strategy drives out $\hat{\lambda}^{\varepsilon}$ for all small enough $\varepsilon>0$.

Corollary 3 Given any completely mixed simple strategy $\lambda^{c}$ and any underdiversified simple strategy $\hat{\lambda}$. Then $\hat{\lambda}^{\varepsilon}$, defined in Corollary 2, is not robust against $\lambda^{c}$-mutants for all sufficiently small $\varepsilon>0$, i.e. the distribution of wealth shares that assigns total wealth to the $\hat{\lambda}^{\varepsilon}$-player is not stable in the population $\left(\hat{\lambda}^{\varepsilon}, \lambda^{c}\right)$.

\section{Appendix}

Proof of Proposition 1. We prove the statement by contraposition. Let $\lambda^{i}, i \in \mathcal{I}$, be a family of adapted trading strategies such that $\lambda^{i}(\omega) \neq \lambda^{j}(\omega)$ for some $i, j \in \mathcal{I}, i \neq j$ on a set $\bar{\Omega}$ of strictly positive $\mathbb{P}$-measure. Let $r \in \Delta^{I}$ with $r^{i} r^{j}>0$. We will show that $r$ cannot be invariant. 
Since $\lambda^{i}, \lambda^{j} \in \operatorname{int} \Delta^{\mathrm{K}}$, and $r^{i} r^{j}>0, \lambda^{i}(\omega) r^{i} \neq \lambda^{j}(\omega) r^{j}$ for all $\omega \in \bar{\Omega}$. This further implies that $a^{i}:=\left(\lambda_{k}^{i} r^{i} / \sum_{l=1}^{I} \lambda_{k}^{j} r^{l}\right)_{k} \neq\left(\lambda_{k}^{j} r^{j} / \sum_{l=1}^{I} \lambda_{k}^{j} r^{l}\right)_{k}=: a^{j}$ for all $\omega \in \bar{\Omega}$, i.e. the 'portfolios' $a^{i}$ and $a^{j}$ are different. Due to the nonredundancy Assumption 4, $f_{i}(\omega, r) \neq f_{j}(\omega, r)$ in equation (7) on a set of strictly positive measure. Hence $r$ is not invariant in the sense of Definition 1.

Proof of Proposition 2. The proof is mainly an application of Oseledets's multiplicative ergodic theorem for random dynamical systems on manifolds, see Arnold (1998, Chapter 4).

The random dynamical system describing the evolution of wealth shares is defined on the simplex $\Delta^{I}$, an $I$-1-dimensional manifold with boundary. We therefore transform the system and consider a conjugate random dynamical system on a subset of the Euclidean space.

Define the projection of the unit simplex,

$$
D^{I-1}:=\left\{y \in \mathbb{R}^{I-1} \mid y_{i} \geq 0, \sum_{i=1}^{I-1} y_{i} \leq 1\right\} \subset \mathbb{R}_{+}^{I-1} .
$$

Further, for each $n \in \mathcal{I}$ define the map

$$
h_{n}: D^{I-1} \rightarrow \Delta^{I}, h_{n}\left(y_{1}, \ldots, y_{I-1}\right):=\left(y_{1}, \ldots, y_{n-1}, 1-\sum_{i=1}^{I-1} y_{i}, y_{n}, \ldots, y_{I-1}\right)
$$

with inverse

$$
h_{n}^{-1}\left(x_{1}, \ldots, x_{I}\right):=\left(x_{1}, \ldots, x_{n-1}, x_{n+1}, \ldots, x_{I}\right) .
$$

$h_{n}$ is a $C^{\infty}$-diffeomorphism. We obtain the conjugate random dynamical system on $D^{I-1}$,

$$
\psi_{n}(t, \omega):=h_{n}^{-1} \circ \phi(t, \omega) \circ h_{n} .
$$

Due to the definition of the space $D^{I-1}$, we can take directional derivatives in the direction of all unit vectors at all points in the interior of $D^{I-1}$ relative to $\mathbb{R}_{+}^{I-1}$. That is we can determine the Jacobian of the conjugate system at all points in $\left\{y \in \mathbb{R}_{+}^{I-1} \mid \sum_{i=1}^{I-1} y_{i}<1\right\}$ (which is an invariant set for the random dynamical system $\psi_{n}$ ).

Note that the origin of $\mathbb{R}^{I-1}$ corresponds to the $n$th corner of the unit simplex. The stability properties of these two fixed points are identical because of the $C^{\infty}$-equivalence of both random dynamical systems.

For notational simplicity we assume without loss of generality that $n=I$. Then the partial derivatives of $\psi_{I}(1, \omega, y)=h_{I}^{-1} \circ \phi(1, \omega) \circ h_{I}(y)$ are given 
by,

$$
\frac{\partial \psi_{I}^{i}(1, \omega, y)}{\partial y_{m}}=-\sum_{k=1}^{K} \frac{R^{k}(\omega)\left(\lambda_{k}^{m}(\omega)-\lambda_{k}^{I}(\omega)\right) \lambda_{k}^{i}(\omega) y_{i}}{\left(\sum_{j=1}^{I-1} \lambda_{k}^{j}(\omega) y_{j}+\lambda_{k}^{I}(\omega)\left(1-\sum_{j=1}^{I-1} y_{j}\right)\right)^{2}}
$$

for all $i \neq m$, and by

$$
\begin{aligned}
\frac{\partial \psi_{I}^{i}(1, \omega, y)}{\partial y_{m}}= & -\sum_{k=1}^{K} \frac{R^{k}(\omega)\left(\lambda_{k}^{m}(\omega)-\lambda_{k}^{I}(\omega)\right) \lambda_{k}^{m}(\omega) y_{m}}{\left(\sum_{j=1}^{I-1} \lambda_{k}^{j}(\omega) y_{j}+\lambda_{k}^{I}(\omega)\left(1-\sum_{j=1}^{I-1} y_{j}\right)\right)^{2}} \\
& +\sum_{k=1}^{K} \frac{R^{k}(\omega) \lambda_{k}^{m}(\omega)}{\sum_{j=1}^{I-1} \lambda_{k}^{j}(\omega) y_{j}+\lambda_{k}^{I}(\omega)\left(1-\sum_{j=1}^{I-1} y_{j}\right)}
\end{aligned}
$$

for all $i=m$.

The stability properties of $e_{I} \in \Delta^{I}$ can be determined by evaluating the Jacobian of $\psi_{I}$ at the origin and applying the multiplicative ergodic theorem of Oseledets. It will be shown that condition (12) resp. (13) ensures that the top Lyapunov exponent of this linear system is strictly negative resp. positive. Results by Wanner (1995), see Arnold (1998, Theorem 7.5.6), ensure that the dynamic behavior of the linearized system carries over (locally) to the nonlinear stochastic system.

From the above expressions, we obtain the Jacobian of $\psi_{I}$ at $y=(0, \ldots, 0)$. It is a diagonal matrix with entry,

$$
A_{m, m}(\omega):=\sum_{k=1}^{K} R^{k}(\omega) \frac{\lambda_{k}^{m}(\omega)}{\lambda_{k}^{I}(\omega)}
$$

The multiplicative ergodic theorem, Arnold (1998, Theorem 4.2.6), implies that the Lyapunov exponents of the fixed point $y=0$ of $\psi_{I}$ are given by $\lim _{T \rightarrow \infty} \frac{1}{T} \ln \left|\prod_{t=0}^{T} A_{m, m}\left(\theta^{t} \omega\right)\right|, m=1, \ldots, I-1$. The integrability condition of the multiplicative ergodic theorem is satisfied because the space $\Delta^{I}$ is compact. By the Birkhoff ergodic theorem, we find that this limit is equal to

$$
\lim _{T \rightarrow \infty} \frac{1}{T} \sum_{t=0}^{T} \ln \left|\sum_{k=1}^{K} R^{k}\left(\theta^{t} \omega\right) \frac{\lambda_{k}^{m}\left(\theta^{t} \omega\right)}{\lambda_{k}^{I}\left(\theta^{t} \omega\right)}\right|=\mathbb{E} \ln \left|\sum_{k=1}^{K} R^{k}(\omega) \frac{\lambda_{k}^{m}(\omega)}{\lambda_{k}^{I}(\omega)}\right|
$$

Zero is a stable fixed point of $\psi_{I}$ if the term in (21) is strictly negative for all $m=1, \ldots, I-1$. If $(21)$ is strictly positive for some $m$, then zero is locally unstable. Due to the diagonal structure of the Jacobian, the eigenspaces correspond to the linear spaces spanned by the unit vectors (restricted to the positive orthant $\mathbb{R}_{+}^{I-1}$ ).

The stability of the original system on $\Delta^{I}$ at the fixed point $e_{I} \in \Delta^{I}$ is determined by the Lyapunov exponents (21). The corresponding eigenspaces 
are given by the vertices. All summands in (21) are positive and we thus have obtained conditions (12) and (13) of the proposition.

Proof of Theorem 1. Obviously, $\lambda^{\star}$ is a completely mixed strategy, i.e. $\sum_{k=1}^{K} \lambda_{k}^{\star}=1$ and $\lambda_{k}^{\star}>0$ for all $k$. Next we define the auxiliary function,

$$
g_{\beta}(\alpha):=\mathbb{E} \ln \left(\sum_{k=1}^{K} R^{k}(\omega) \frac{\alpha_{k}}{\beta_{k}}\right)
$$

in accordance with Proposition 2. For each fixed strategy $\beta \in \operatorname{int} \Delta^{\mathrm{K}} \subset \mathbb{R}^{\mathrm{K}}$, $g_{\beta}: \operatorname{int} \Delta^{\mathrm{K}} \rightarrow \mathbb{R} . g_{\beta}(\alpha)$ is the Lyapunov exponent of the distribution of wealth that assigns total wealth to the 'status quo' population that plays strategy $\beta$ in a market in which $\alpha$ is the only the alternative strategy.

By Proposition 2 the first assertion of the theorem follows if we can show that $g_{\lambda^{\star}}(\alpha)<0$ for all $\alpha \in \operatorname{int} \Delta^{\mathrm{K}}$ with $\alpha \neq \lambda^{\star}$.

We prove that $g_{\beta}(\alpha)$ is strictly concave for all $\beta \in$ int $\Delta^{\mathrm{K}}$ and that $g_{\lambda^{\star}}(\alpha)$ takes on its maximum value at $\alpha=\lambda^{\star}$.

To ensure strict concavity it suffices to show that $\alpha \mapsto g_{\beta}(\alpha)$ is strictly concave on the space $\mathbb{R}_{++}^{K}$, because restriction of the domain to the linear subspace int $\Delta^{\mathrm{K}}$ preserves strict concavity. The function $\ln \sum_{k=1}^{K}\left(R^{k}(\omega) \alpha_{k} / \beta_{k}\right)$ is concave for all $\omega$ and-due to the no-redundancy Assumption 4-strictly concave on a set of positive measure. Therefore $g_{\beta}(\alpha)$ is strictly concave for each fixed $\beta \in \operatorname{int} \Delta^{\mathrm{K}}$.

We can now employ that $\lambda^{\star}$ is the unique maximum of $g_{\lambda^{\star}}(\alpha)$ on int $\Delta^{\mathrm{K}}$ if all directional derivatives at this point are zero.

The partial derivative of $g_{\beta}(\alpha)$ with respect to the $i$-th component $\alpha_{i}$ is given by

$$
\frac{\partial g_{\beta}(\alpha)}{\partial \alpha_{i}}=\mathbb{E} \frac{R^{i}(\omega) / \beta_{i}}{\sum_{k=1}^{K} R^{k}(\omega) \frac{\alpha_{k}}{\beta_{k}}}
$$

Observe that interchanging integration and differentiation is allowed because $\ln \left(\sum_{k=1}^{K} R^{k}(\omega) \alpha_{k} / \beta_{k}\right)$ is integrable for each fixed $\alpha$ (follows from $\mathbb{E} R^{k}(\omega) \leq$ $1<\infty$ for all $k$ ) and $\mathbb{E}\left(R^{i}(\omega) / \sum_{k=1}^{K} R^{k}(\omega)\right) \leq 1<\infty$ (this follows from the fact that $R^{k}(\omega) \geq 0$ for all $k$ and all $\omega$ by assumption). The last equation implies

$$
\frac{\partial g_{\lambda^{\star}}\left(\lambda^{\star}\right)}{\partial \alpha_{i}}=\mathbb{E} \frac{R^{i}(\omega)}{\lambda_{i}^{\star}}=\mathbb{E} \frac{R^{i}(\omega)}{\mathbb{E} R^{i}} \equiv 1
$$

for all $i=1, \ldots, K$, since $\sum_{k=1}^{K} R^{k}(\omega) \equiv 1$ for all $\omega$. 
The directional derivative of $g_{\lambda^{\star}}$ in the direction $\left(d \alpha_{1}, \ldots, d \alpha_{K}\right)$ with the restriction $\sum_{k=1}^{K} d \alpha_{k}=0$ (which is a vector in the simplex) is equated as

$$
\sum_{i=1}^{K} \frac{\partial g_{\lambda^{\star}}\left(\lambda^{\star}\right)}{\partial \alpha_{i}} d \alpha_{i}=0
$$

Corollary 1 ensures that any portfolio rule different to $\lambda^{\star}$ is not evolutionary stable.

Let us next prove that any strategy $\beta \neq \lambda^{\star}$ with $\beta \in$ int $\Delta^{\mathrm{K}}$ is not locally evolutionary stable. Since - due to the i.i.d. assumption - the dependence of the strategy $\beta$ on the past $\omega^{-1}$ does not affect the expected value in Proposition 2 , cf. equation (16), we can restrict our analysis to simple strategies $\beta$. A strategy $\beta \neq \lambda^{\star}$ is not locally evolutionary stable, if for any neighborhood of $\beta$ there exists an $\alpha$ such that $g_{\beta}(\alpha)>0$. It suffices to show that the directional derivative of $g_{\beta}$ at $\beta$ is strictly positive in one direction.

Since $\beta \neq \lambda^{\star}$ and both are points in the simplex there exists $i \neq j$ with $\beta_{i}>\lambda_{i}^{\star}$ and $\beta_{j}<\lambda_{j}^{\star}$. Note that we have assumed a minimum of two assets.

The directional derivative of $g_{\beta}$ at $\beta$ in the direction $d \alpha$ given by $d \alpha_{i}=$ $-1 / 2, d \alpha_{j}=1 / 2$, and zero otherwise, is given by,

$$
\sum_{k=1}^{K} \frac{\partial g_{\beta}(\beta)}{\partial \alpha_{k}} d \alpha_{k}=\sum_{k=1}^{K} \frac{\mathbb{E} R^{k}}{\beta_{k}} d \alpha_{k}=\frac{1}{2}\left(\frac{\lambda_{j}^{\star}}{\beta_{j}}-\frac{\lambda_{i}^{\star}}{\beta_{i}}\right)>0 .
$$

Proof of Theorem 2. This result follows mainly from an application of the proof of Theorem 1. For any adapted strategy $\lambda^{i}(\omega)=\lambda^{i}\left(\omega^{-1}\right)$ with $\lambda^{i}(\omega) \neq \lambda^{\star}$ on a set $\bar{\Omega}$ of strictly positive $\mathbb{P}$-measure we have the following. Fix any $\omega^{-1} \in S^{\mathbb{N}}$. Then $g_{\lambda^{\star}}\left(\lambda^{i}\left(\omega^{-1}\right)\right) \leq 0$ for all $\omega$ and the inequality is strict for all $\omega \in \bar{\Omega}$, cf. proof of Theorem 1. This implies that condition (i) in Proposition 2 holds. Therefore $\lambda^{\star}$ is evolutionary stable.

To prove that no other adapted strategy can be evolutionary stable, fix any adapted strategy $\lambda^{i}(\omega)=\lambda^{i}\left(\omega^{-1}\right)$ with $\lambda^{i}(\omega) \neq \lambda^{\star}$ on a set $\bar{\Omega}$ with $\mathbb{P}(\bar{\Omega})>0$.

For every fixed $\omega^{-1} \in S^{\mathbb{N}}$, we employ the procedure that has been applied in proof of Theorem 1 to find a strategy such that for a strategy, say $\lambda^{j}$, $g_{\lambda^{i}\left(\omega^{-1}\right)}\left(\lambda^{j}\right) \geq 0$ where the inequality is strict for all $\omega \in \bar{\Omega}$.

If $\mathcal{S}$ is the power set of $S$, then $\mathcal{F}$ is the power set of $S^{\mathbb{Z}}$. Therefore the strategy $\lambda^{j}(\omega):=\lambda^{j}\left(\omega^{-1}\right)$ is measurable. By construction it is also adapted and in any prescribed neighborhood of $\lambda^{i}$. We have defined $\lambda^{j}$ in the way 
that condition (ii) in Proposition 2 holds for the pair of strategies $\lambda^{i}, \lambda^{n}=\lambda^{j}$. As a result the adapted strategy $\lambda^{i}$ is not evolutionary stable.

Proof of Corollary 2. According to Proposition 2(i) it suffices to show that

$$
\mathbb{E} \ln \left(\sum_{k: \hat{\lambda}_{k}>0}^{K} R^{k}(\omega) \frac{\hat{\lambda}_{k}}{(1-\varepsilon) \hat{\lambda}_{k}+\varepsilon / S}\right)<0
$$

for all small $\varepsilon>0$. The left-hand side of this equation is strictly increased by omitting $\varepsilon / S$ in the denominator. We thus obtain the sufficient condition,

$$
\mathbb{E} \ln \left(\sum_{k: \hat{\lambda}_{k}>0} R^{k}(\omega)\right) \leq \ln (1-\varepsilon)
$$

Since there is at least one $k$ such that $\hat{\lambda}_{k}=0$, we find that $\sum_{k: \hat{\lambda}_{k}>0} R^{k}(\omega)<1$ on a set of positive measure (the term is bounded by 1 for all $\omega$ ), the lefthand side of $(23) \mathbb{E} \ln \left(\sum_{k: \hat{\lambda}_{k}>0} R^{k}(\omega)\right)<0$. Therefore $(23)$ is satisfied for all small enough $\varepsilon$.

Proof of Corollary 3. Again we employ Proposition 2. The local instability result says that the assertion of the Corollary is true, if

$$
\mathbb{E} \ln \left(\sum_{k=1}^{K} R^{k}(\omega) \frac{\lambda_{k}^{c}}{(1-\varepsilon) \hat{\lambda}_{k}+\varepsilon / S}\right)>0
$$

for all small $\varepsilon>0$.

Noting that

$$
\sum_{k=1}^{K} \frac{R^{k}(\omega) \lambda_{k}^{c}}{(1-\varepsilon) \hat{\lambda}_{k}+\varepsilon / S}=\sum_{k: \hat{\lambda}_{k}>0} \frac{R^{k}(\omega) \lambda_{k}^{c}}{(1-\varepsilon) \hat{\lambda}_{k}+\varepsilon / S}+\sum_{k: \hat{\lambda}_{k}=0} R^{k}(\omega) \frac{S \lambda_{k}^{c}}{\varepsilon}
$$

$\lambda_{k}^{c}>0$ for all $k$, and $R^{k}(\omega)>0$ on a set of positive measure for all $k$ with $\hat{\lambda}_{k}=0$, we find that the left-hand side of (24) tends to infinity as $\varepsilon \rightarrow 0$.

\section{References}

Algoet, P. H., And T. M. Cover (1988): "Asymptotic Optimality and Asymptotic Equipartition Properties of Log-Optimum Investment," $A n$ nals of Probability, 16, 876-898. 
Arnold, L. (1998): Random Dynamical Systems. Springer-Verlag, Berlin.

Blume, L., And D. Easley (1992): "Evolution and Market Behavior," Journal of Economic Theory, 58, 9-40. (2000): "If You're So Smart, Why Aren't You Rich? Belief Selection in Complete and Incomplete Markets," mimeo, Department of Economics, Cornell University.

Breiman, L. (1961): "Optimal gambling systems for favorable games," Fourth Berkeley Symposium on Mathematical Statistics and Probability, $1,65-78$.

Cover, T. (1984): "An algorithm for maximizing expected log-investment return," IEEE Transformation Information Theory, 30, 369-373.

(1991): "Universal Portfolios," Mathematical Finance, 1, 1-29.

HaKansson, N. (1970): "Optimal investment and consumption strategies under risk for a class of utility functions," Econometrica, 38, 587-607.

Karatzas, I., And S. Shreve (1998): Methods of Mathematical Finance. Springer-Verlag, New York.

Kelly, J. (1956): "A New Interpretation of Information Rate," Bell System Technical Journal, 35, 917-926.

Maynard Smith, J., and G. Price (1973): "The Logic of Animal Conflict," Nature, 246, 15-18.

SAndroni, A. (2000): "Do Markets Favor Agents Able to Make Accurate Predictions?," Econometrica, 68, 1303-1341.

Schenk-Hoppé, K. R. (2001): "Random Dynamical Systems in Economics," Stochastics and Dynamics, 1, 63-83.

SciubBA, E. (1998): "The Evolution of Portfolio Rules and the Capital Asset Pricing Model," Working Paper, University of Cambridge, mimeo.

Thorp, E. (1971): "Portfolio choice and the Kelly criterion," In Stochastic Models in Finance,W.T. Ziemba and R.G. Vickson, eds., 599-619.

Wanner, T. (1995): "Linearization of random dynamical systems," in $D y$ namics Reported Vol 4, ed. by C. Jones, U. Kirchgraber, and H. O. Walther, pp. 203-269. Springer-Verlag. 